\title{
CLINICAL COMPARISON OF TOPICAL APPLICATION OF PLATELET RICH FIBRIN VERSUS HYALURONIC ACID GEL IN PAIN MANAGEMENT AND WOUND HEALING AFTER FREE GINGIVAL GRAFT HARVESTING: A RANDOMIZED CONTROLLED CLINICAL TRIAL
}

\author{
Mostafa Soliman*, Ahmed El-Barbary ${ }^{* *}$, Weam Elbattawy ${ }^{* * *}$ and Mona Shoeib ${ }^{* * * *}$
}

\begin{abstract}
Aim: This study aimed to compare the ability of platelet rich fibrin (PRF) versus hyaluronic acid (HA) in decreasing the postoperative pain and promoting healing in the palatal wound following harvesting free gingival graft (FGG).
\end{abstract}

Methods: 26 patients with narrow zone of keratinized tissue seeking for orthodontic treatment requiring FGG for soft tissue augmentation were randomly assigned into two equal groups; HA group where the palatal wound was covered by $0.2 \% \mathrm{HA}$ gel or PRF group where the palatal wound was covered by PRF. Both were then covered by non-eugenol periodontal pack. Assessment of postoperative pain for both groups was recorded using the Numerical Pain Score (NPS) (1-10) for a period of 7 days. Healing of the palatal wound was assessed using Landry Healing Index at day $3,1^{\text {st }}, 2^{\text {nd }}, 3^{\text {rd }}$ weeks and 42 days.

Results: Post-operative pain assessed using the NPS showed no statistically significant difference between HA and PRF groups in all time intervals. All patients had reduction in postoperative pain following the harvesting of FGG after 1 week. For the palatal wound healing, there was a statistically significant difference between the two treatment modalities at week 1 in favor for PRF group ( $\mathrm{P}=0.002)$, while at day 3, week 2, week 3 and 42 days, there was no statistically significant difference between the two groups $(\mathrm{P}=0.678,0.088,0.155$ and 0.460$)$ as both of them improved the healing of the palatal wound.

Conclusion: Both treatment modalities $(0.2 \% \mathrm{HA}$ gel and PRF) reduced the post-operative pain and improved the palatal wound healing following harvesting of FGG.

Conflict of interest and source of funding statement: The authors declare that they have no conflict of interest. The study was self-funded

KEYWORDS: Hyaluronic Acid, platelet rich fibrin, free gingival graft, numerical pain scale.

\footnotetext{
* M.Sc. of Periodontology, Department of Oral Medicine and Periodontology, Faculty of Dentistry, Cairo University. ** Associate professor, Department of Oral Medicine and Periodontology, Faculty of Dentistry, Cairo University. ***Associate professor, Department of Oral Medicine and Periodontology, Faculty of Dentistry, Cairo University. ***** Professor, Department of Oral Medicine and Periodontology, Faculty of Dentistry, Cairo University.
} 


\section{INTRODUCTION}

A soft tissue graft is the withdrawal of soft tissue that is completely detached from its original donor site and placed in a prepared recipient bed (American Academy of Periodontology 2001). Many intra-oral sites are available for harvesting of soft tissue graft, the hard palate is the most frequent donor site for connective tissue graft (CTG) and free gingival grafts (FGG). The aim of soft tissue grafting may be for augmenting the zone of keratinized tissue around teeth or dental implants, increasing tissue thickness and recession coverage.

\section{(Zucchelli et al., 2010).}

Soft tissue grafts are generally classified into autogenous grafts and allograft (Zucchelli et al., 2010). The autogenous grafts are soft tissue grafts containing the vital cells of patient himself, therefore, when used to correct recession defects, clinicians may anticipate creeping attachment which is not preset in the allograft. Autogenous grafts are subdivided into pedicle grafts that maintain their source of the blood supply and free grafts that are deprived from their blood supply. Free grafts include the epithelialized free gingival graft (FGG), deepithelialized free gingival graft and connective tissue grafts (CTG). The key of survival of these free grafts is presence of sufficient areas of the grafts in close contact with the recipient bed for establishment of revascularization between the bed and the preexisting vessels in the graft (Silva et al, 2007).

The main indication of FGG is to reestablish an adequate keratinized tissue width and thickness in the presence of mucogingival defects (Zucchelli 2015). The long-term efficacy of an FGG compared with contralateral untreated sites has been assessed by Agudio et al., (2016) that observed the stability of the gingival margin and the prevention of gingival recessions after augmentation by FGG compared to untreated contralateral sites which associated with increased recession depth. There are many risk factors determine the outcomes of FGG such as improper preparation of the recipient site, inadequate graft size and thickness, poor adaptation to the recipient bed and failure to stabilize the graft.

However, many drawbacks are associated with FGG, such as the significant shrinkage (around 30\%) that occurs during the healing process, increased surgical time, poor color match with the surrounding tissue, the malalignment of mucogingival junction and higher patient morbidity as the donor site heals by secondary intention (de Resende et al., 2019). Many clinical trials aimed to reduce the postoperative pain following harvesting FGG using low level laser (diode laser) (Ozcelik et al, 2016), topical simvastatin (Madi et al, 2017), platelet-rich fibrin (Bahammam, 2018), application of moist exposed wound ointment (MEBO and Hyaluronic acid )( Hassan et al,2021).

Hyaluronic acid is a high molecular weight nonsulphated polysaccharide that is one of component of the glycosaminoglycan's family. It was found in various body fluids such as synovial fluid, serum, saliva and gingival crevicular fluid and it represents a major component of the extracellular matrix of skin and connective tissue of all periodontal tissues. One of the major features of HA is the hygroscopicity that allows it to maintain the conformational stiffness to retain the water and the viscoelasticity which provides stability and elasticity to tissues and delay the penetration of bacteria. In the market, it is available as a topical application form in 2 concentrations; $0.2 \%$ and $0.8 \%$ which are highly biocompatible, non-immunogenic, bacteriostatic, anti-inflammatory, anti-edematous and promote healing of the soft tissues (Yildirim et al., 2017).

PRF is a platelet concentrate that consists of an autologous leukocyte platelet-rich fibrin matrix. PRF is composed of a 3 dimensional molecular structure rich in cytokines, platelets, and stem cells. PRF acts as a biodegradable scaffold that favors the development of microvascularization and guides the 
migration of epithelial cells to the wound surface. Also, PRF seems to have an ability to sustain the release of its growth factors up to 4 weeks, thus stimulating the environment for an optimum wound healing. PRF has been reported as a good healing biomaterial with great potential for soft tissue regeneration without the undersirable inflammatory reactions (Borie et al., 2015).

HA and PRF could achieve a high percentage of reduction in postoperative pain, and evidence has supported the role of using hyaluronic acid or PRF in decreasing the postoperative pain (Sousa et al., 2020 ;Hassan et al., 2021). However, scarce evidence exists regarding the superiority of a specific material over the other. Therefore, this study aimed to compare between both materials in reducing postoperative pain and enhancing healing in the palatal wound after harvesting FGG.

\section{MATERIALS AND METHODS}

\section{Study design}

This study was designed as a parallel, randomized, controlled clinical trial to compare the clinical outcome of $0.2 \%$ hyaluronic acid gel versus PRF in decreasing the post-operative pain and promoting the healing of the palatal wound after harvesting the free gingival graft (FGG). The study protocol was registered in Clinical Trials.gov (ID: NCT03814707) and approved by The Research Ethics committee, Faculty of Dentistry, Cairo University (February 2019). This study was conducted according to Helsinki declaration (1975) revised in 2013 and the detailed operation and follow up periods were clearly described in details to all patients selected in this clinical trial. All subjects participated in this trial, signed a written consent and fully agreed to participate in this work. This trial was re- ported according to CONSORT guidelines. (Schulz et al., 2010)

\section{Study population}

This investigation included 26 patients (10 males and 16 females, aged 20 to 40 years) having a mucogingival problem that required gingival augmentation by FGG. Subjects were selected from the outpatient clinic, Department of Oral Medicine and Periodontology, Faculty of Dentistry, Cairo University between April 2019 and January 2020 according to the following inclusion criteria: 1) patients with mucogingival defects requiring augmentation with a FGG from the palate, 2) patients able to tolerate surgical periodontal procedures and ready to be compliant to oral hygiene instructions, 3) patients who provided an informed consent and 4) patients who had no previous periodontal surgery in the experimental sites. While those who were excluded from the study were 1) patients with any systemic diseases or medications that might be a contraindication for any surgeries and could compromise wound healing, 2) loss of maxillary premolars and molars, 3) uncooperative patients, 4) pregnant or nursing women and 5) smokers.

\section{Pretreatment phase}

Full medical history and proper diagnosis of the mucogingival defect were performed to decide the suitable treatment plan and to ensure that the patient was fulfilling the inclusion criteria. After the patient was accepted to be enrolled in this study, full mouth supra and subgingival debridement was performed using ultrasonic device* with supragingival scaling inserts ${ }^{* *}$ followed by Gracey curettes *** for proper subgingival debridement if needed. Patient preparation was completed in a single visit. Mechanical plaque control was applied involving

\footnotetext{
* Woodpecker UDS-P with LED, China.

** EMS Woodpecker ultrasonic scaler tip, Woodpecker, China.

*** HuFriedy Gracey's curette; HuFriedy, Chicago, USA.
} 
proper oral hygiene instructions and tooth brushing twice daily by soft toothbrush using modified bass technique. Mouth rinse with $0.12 \%$ chlorhexidine* was prescribed twice daily for 2 weeks.

\section{Randomization}

A computer-generated random allocation sequence was executed by external assistant who was not involved in the recruitment. Allocation concealment was achieved by sequentially numbered, opaque, sealed envelopes including the randomization code for each patient.

\section{Blinding}

The current trial was a single-blinded clinical trial. Blinding included the outcome assessor and the statistician since it was impossible for the operator and the participants to be blinded due to the different nature of the two interventions.

\section{Sample size calculation}

Based on a previous study by Femminella, (2016), the difference in pain scores between the two groups was $2.3 \pm 2$. Using power $80 \%$ and $5 \%$ significance level, 13 participants in each group were sufficient to detect this difference. This number has been increased to 15 to compensate any possible loss of any patient during the treatment period. Sample size calculation was achieved using PS program**.

\section{Patient reported outcomes (postoperative pain):}

Patient reported quality of life related outcomes have been assessed in the form of post-operative pain. It was assessed by using Numerical pain scale (NPS) described by Sirintawat et al. (2017). NPS was reported by the patients with the numbers from
0 to 10 , where the scale ' 0 ' represented 'no pain' and scale ' 10 ' represented the 'severe pain'. The NPS was given to the patients in written form and the participants were asked to fill this form daily from day 1 to day 7 post-surgically.

\section{Clinical parameters (soft tissue healing of the palatal wound):}

Clinical parameters were assessed by evaluating the soft tissue healing of the palatal wound at the donor site using Landry Healing Index (1988). The parameters included bleeding upon palpation, presence of granulation tissues, presence of necrotic tissues and epithelization of the palatal wound. These parameters were reported at day 3 , week 1 , week 2 , week $3 \&$ after 42 days postoperatively.

\section{Treatment protocol:}

\section{Preparation of recipient site:}

In both groups, preparation of the recipient site was performed according to Sullivan (1968) as the horizontal incision was performed at the level of mucogingival junction this was followed by two vertical releasing incisions performed at the mesial and distal ends of the horizontal incision and extended beyond the mucogingival line. A splitthickness flap was elevated, and the dissection was extended in the apical direction about $7 \mathrm{~mm}$. The flap was excised at the apical end with surgical scissors. The dimension of the recipient site was determined by using a sterile aluminum foil adapted properly on the recipient bed and acted as a template to get the actual size of the desired FGG to be harvested from the palate.

\section{Harvesting of Free gingival graft (FGG):}

Two horizontal incisions were performed, where

\footnotetext{
* Hexitol: Chlorhexidine HCL mouthwash, The Arab Drug Company for pharmaceutical \& CHEM. IND. CO. CairoEgypt.

** Power and sample size program: biostat.mc.vanderbilt.edu/twiki/bin/view/Main/PowerSampleSize
} 
the coronal incision was performed at least $2 \mathrm{~mm}$ apical to the gingival margin of the adjacent teeth and two vertical incisions were traced to delineate the area to be grafted. The thickness of the graft was maintained uniform $2.5 \mathrm{~mm}$ while proceeding apically with the blade. Care was taken to avoid removing of the palatal periosteum (Zucchelli et al. 2010).

\section{Hyaluronic acid group}

After harvesting the FGG, the bleeding was controlled by application of pressure using sterile gauze for 5 minutes followed by application of $0.2 \%$ Hyaluronic acid gel (Gengigel)* using sterile plastic syringe. The palatal wound was immediately covered by non-eugenol periodontal pack (Pericem) ${ }^{* *}$ and stabilized by criss-cross suturing technique using 4-0 Prolene ${ }^{* * *}$ suture material anchored between the contact areas. Three days postoperatively, the patients were recalled, and the periodontal pack was removed for evaluating the healing of the palatal wound. Then $0.2 \%$ HA gel was re-applied again, and the palatal donor site was repacked with periodontal pack. The periodontal pack was finally removed after 1 week and evaluation of the healing of the palatal wound was done.

\section{PRF group}

Platelet rich fibrin (PRF) was prepared by drawing $10 \mathrm{ml}$ of venous blood from the antecubital vein using butterfly needle then the blood was immediately transferred to two glass-coated plastic tubes without anticoagulant. The blood sample was immediately centrifuged in an electric centrifuge at $3000 \mathrm{rpm}$ for $10 \mathrm{~min}$ (G-force of $3000 \mathrm{rpm}$ equivalent to $400 \mathrm{gm}$ ) (Choukroun, 2001). The middle part of PRF layer was separated and collected to be used over the palatal donor site. The PRF membranes were placed in double layers and sutured by crisscross suturing technique using 4-0 Prolene suture material. The palatal wound was then covered by non-eugenol periodontal pack without anchoring it to avoid excessive trauma for the palatal soft tissue that may affect the intensity of the postoperative pain of the study . After three days post surgically, patients were recalled, and the periodontal pack was removed for evaluation of the palatal wound healing. The palatal wound was then repacked using non-eugenol periodontal dressing. The Periodontal pack was removed after one week and the clinical photographs were taken for the palatal wounds in both groups at day 3, after 1 week, 2 weeks, 3 weeks and 42 days for evaluation of the healing of the palatal wound.
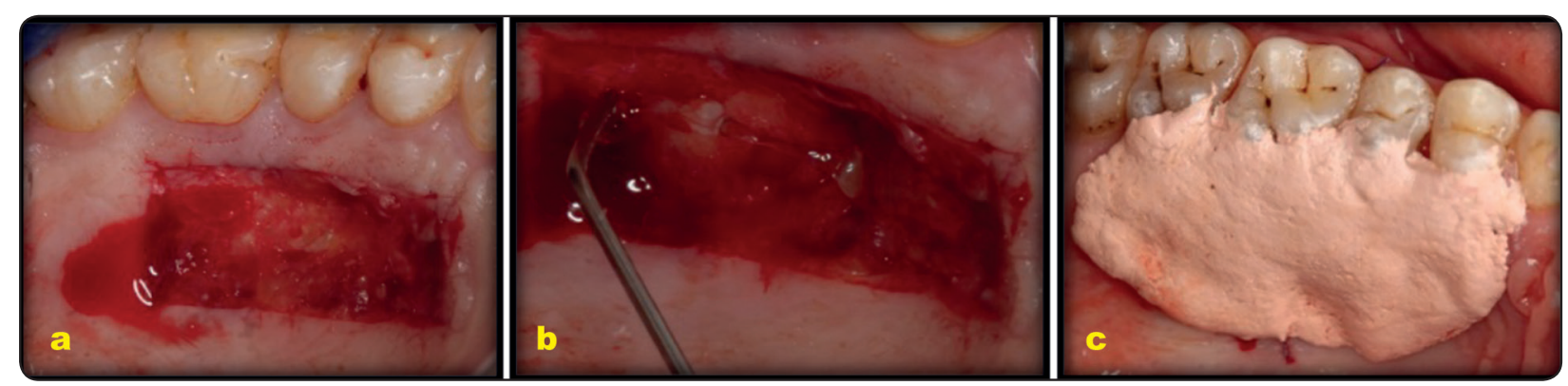

Fig. (1) Represents a case of hyaluronic acid group (a) palatal wound after harvesting FGG.(b) application of hyaluronic acid gel, (c) application of periodontal dressing to stabilize the HA gel .

\footnotetext{
* Gengigel ${ }^{\circledR}$ Ricerfarma S.r.l., Milano, Italy

** Pericem cement quengco Non eugenol-Italy

*** Citek-PM92030f4 Manfuctring by Huaiyin china
} 

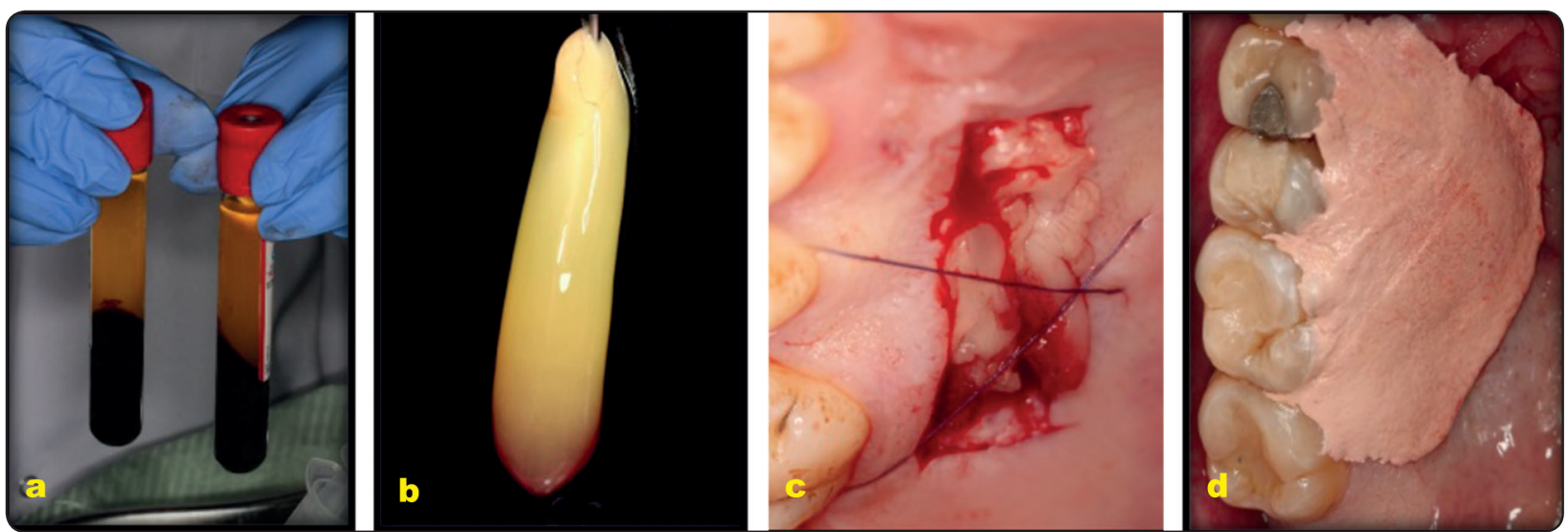

Fig. (2) Represents a case of PRF group (a) PRF immediately after centrifugation (b) A strongly polymerized acellular layer. (c) PRF stabilized over the palatal wound (d) periodontal dressing covering the PRF.

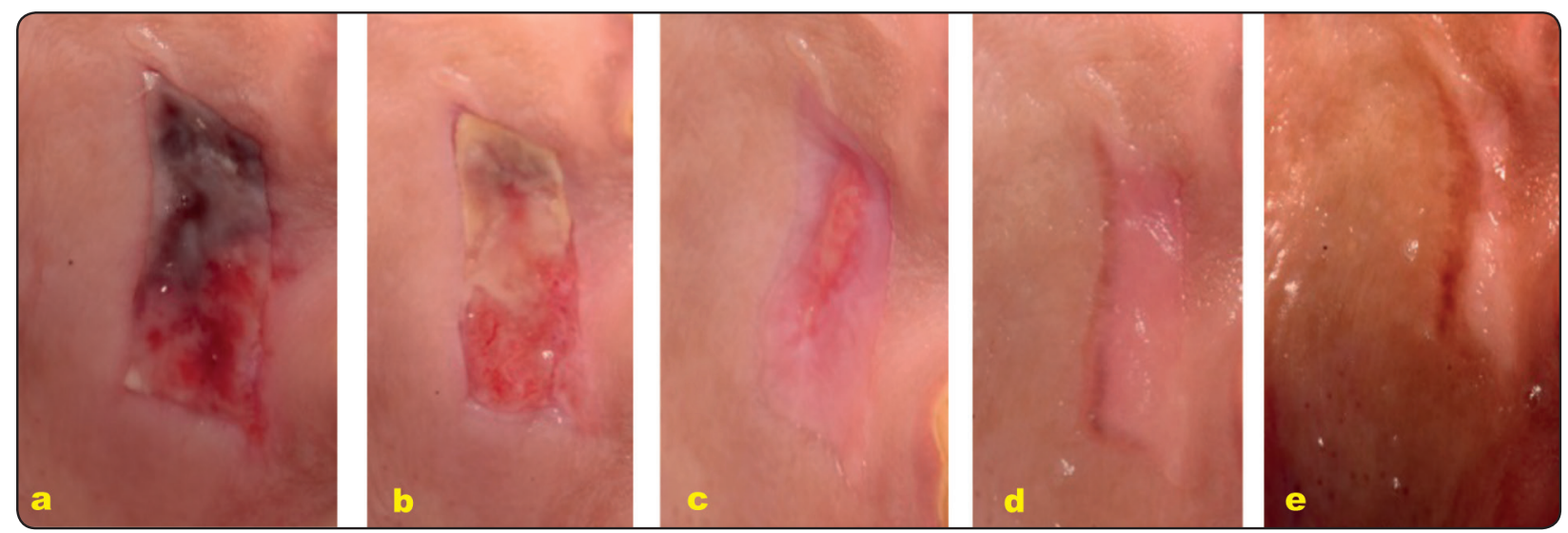

Fig. (3) Healing of the palatal wound in HA group at (a) 3 days, (b) 1 week, (c) 2 weeks, (d) 3 weeks, (e) 42 days
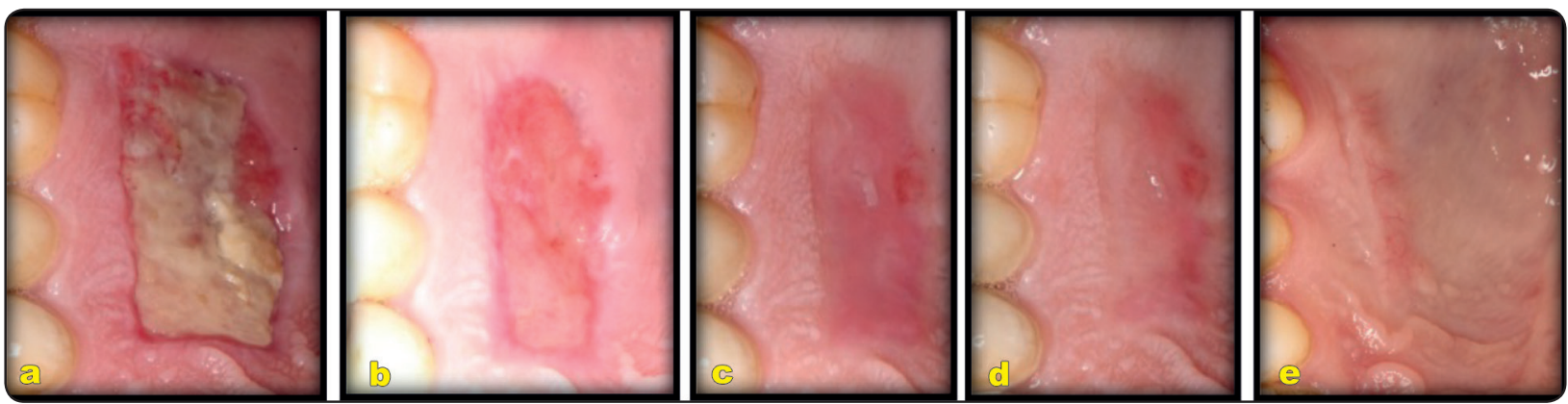

Fig. (4) Healing of the palatal wound in PRF group at (a) 3 days, (b) 1 week, (c) 2 weeks, (d) 3 weeks, (e) 42 days 


\section{Post-surgical phase}

Systemic antibiotics were prescribed for every patient (Amoxicillin $500 \mathrm{mg}$ t.i.d) * orally for 5 days post surgically to prevent the postsurgical infection and the pain was controlled at the day of the surgery by prescribing (Ibuprofen $600 \mathrm{mg}$ two times) ${ }^{* *}$ at the day of the surgery while no analgesics were prescribed after the day of the surgery. Patients were advised to rinse with antiseptic mouth rinse (0.12\% Chlorhexidine HCL) Hexitol twice daily for 1 minute for a period of two weeks after the surgery. Patients were instructed to avoid any hard brushing and trauma to the surgical site for 2 weeks (Zucchelli et al., 2010; Yıldırım et al., 2017).

\section{Statistical analysis}

The median and range values were calculated for each group in each test. Data were explored for normality using Kolmogorov-Smirnov and Shapiro-Wilk tests, the post-operative pain and healing of the palatal wound showed non-parametric (not-normal) distribution. Mann Whitney test was used to compare between two groups in non-related samples. while Wilcoxon test was used to compare between two groups in related samples. The significance level was set at $\mathrm{P} \leq 0.05$. Statistical analysis was performed with IBM ${ }^{\circledR}$ SPSS ${ }^{\circledR}$ Statistics Version 20 for Windows.

\section{RESULTS}

\section{Postoperative pain}

In the hyaluronic acid group, the changes in median (range) of postoperative pain reported by patients treated with $0.2 \% \mathrm{HA}$ gel as a palatal dressing on a Numerical pain scale (NPS) is represented in Table (1). The calculated median (range) of pain was $1(0-10), 5(0-10), 4(0-10), 3$ $(0-10), 1(0-10), 1(0-10)$ and $0(0-10)$ at days 1,2 ,
$3,4,5,6$ and 7 respectively with the highest pain score found in day 2 with a median (range) of 5 (010 ), while the least pain score was found in day 7 with a median (range) of 0 (0-10). Although there was a decrease in the postoperative pain scores after 7 days for all subjects, yet there was no statistically significant difference in pain scores $(\mathrm{P}=0.485)$ between days $1,2,3,4,5,6$ and 7 .

The changes in median (range) of postoperative pain reported by patients treated with PRF as a palatal dressing on a Numerical pain scale (NPS) is represented in Table (1). The calculated median (range) of pain was 1 (0-10), $2(0-10), 4(0-10), 3$ (0-10), 2 (0-10), 0 (0-10), 0 (0-6) at days 1, 2, 3, 4, 5,6 and 7 respectively with the highest pain score found in day 3 as median (range) 4 (0-10), while the least pain score was found in days 6 and 7 as median (range) 0 (0-10) and $0(0-6)$ respectively. The pain scores were reduced from day 1 to day 7 postoperatively, however there was no statistically significant difference in pain score $(\mathrm{P}=0.206)$ between day 1, 2, 3, 4, 5, 6 and 7 .

On comparing both groups over the study period, it was found that the reported pain score in day 2 and day 6 showed higher pain score in HA group than the PRF group with median (range) 5(010) and 1(0-10) for the HA group versus $2(0-10)$ and $0(0-10)$ for the PRF group respectively with no statistically significant difference between both groups $(\mathrm{P}=0.380)$ at day 2 and at day $6(\mathrm{P}=0.603)$. On the other hand, the reported pain score at day 5 was higher in PRF group than HA acid group with median (range) 2 (0-10) versus $1(0-10)$ respectively but also with no statistically significant difference $(\mathrm{P}=0.682)$. Therefore, there was no statistically significant difference in pain scores between the two studied groups at all time intervals from day 1 to day 7 postoperatively with $\mathrm{P}$-value $(\mathrm{P}=0.534,0.380$, $0.704,0.777,0.682,0.603$ and 0.474$)$ respectively.

\footnotetext{
* E-mox 500 mg Cap., E.I.P.I.CO., Egyptian Int. Pharmaceutical Industrial Co., A.R.E

** Ibuprofen $600 \mathrm{mg}$, Abbott, Egypt.
} 


\section{Soft Tissue Healing of the Palatal Wound}

The changes in the median (range) values of the soft tissue healing of the palatal wound at the donor site after the use of $0.2 \%$ HA gel are presented in Table (2). The healing scores at day 3, week 1, week 2 , week 3 and day 42 are 2 (1-3), 2 (1-3), 3 (2$5), 4$ (2-5) and 5 (2-5) respectively. Although there was no difference between day 3 and week 1 , the results presented showed a statistically significant difference in the healing of the palatal wound between (day 3 , week 1), weeks 2, 3 and day 42 post- operatively with $(\mathrm{P}<0.001)$.

The changes in the median (range) values of the soft tissue healing of the palatal wound at the donor site are presented in Table (2). The healing scores at day 3 , week 1 , week 2 , week 3 and day 42 are 2 (12), 3 (2-4), 3 (2-4), 4 (3-5) and 5 (3-5) respectively. Although there was no difference between week 1 and week 2 , the results presented showed a statistically significant difference in the healing of the palatal wound between (weeks 1,2 ), day 3 , weeks 3 and 4 and 42 days postoperatively with $(\mathrm{P}<0.001)$.

TABLE (1) Changes in postoperative pain at 7 days postsurgically in both groups.

\begin{tabular}{cccccc}
\hline Period & $\begin{array}{c}\text { Pain in HA Group } \\
\text { Median (Range) }\end{array}$ & $\begin{array}{c}\text { Pain in PRF Group } \\
\text { Median (Range) }\end{array}$ & Z-Value & P-Value & Effect size \\
\hline Day 1 & $1(0-10)$ & $(0-10) 1$ & 0.621 & $0.534 \mathrm{~ns}$ & 0.117 \\
\hline Day 2 & $5(0-10)$ & $(0-10) 2$ & 0.878 & $0.380 \mathrm{~ns}$ & 0.166 \\
\hline Day 3 & $4(0-10)$ & $(0-10) 4$ & 0.379 & $0.704 \mathrm{~ns}$ & 0.072 \\
\hline Day 4 & $3(0-10)$ & $(0-10) 3$ & 0.283 & $0.777 \mathrm{~ns}$ & 0.053 \\
\hline Day 5 & $1(0-10)$ & $(0-10) 2$ & 0.410 & $0.682 \mathrm{~ns}$ & 0.078 \\
\hline Day 6 & $1(0-10)$ & $(0-10) 0$ & 0.520 & $0.603 \mathrm{~ns}$ & 0.098 \\
\hline Day 7 & $0(0-10)$ & $(0-6) 0$ & 0.717 & $0.474 \mathrm{~ns}$ & 0.135 \\
\hline p-value & 0.485 & 0.206 & &
\end{tabular}

ns; non-significant $(p>0.05)$

TABLE (2) Changes in the healing of the palatal wound for both groups at day3, week1, week 2 week 3 and 42 days.

\begin{tabular}{cccccc}
\hline Period & $\begin{array}{c}\text { Healing in HA Group } \\
\text { Median (Range) }\end{array}$ & $\begin{array}{c}\text { Healing in PRF Group } \\
\text { Median (Range) }\end{array}$ & Z-Value & P-Value & Effect size \\
\hline Day 3 & $2(1-3)^{\mathrm{a}}$ & ${ }^{\mathrm{a}}(1-3) 2$ & 0.415 & $0.678 \mathrm{~ns}$ & 0.078 \\
\hline Week 1 & $2(1-3)^{\mathrm{a}}$ & ${ }^{\mathrm{b}}(1-3) 3$ & 3.111 & $0.002^{*}$ & 0.588 \\
\hline Week 2 & $3(2-5)^{\mathrm{b}}$ & ${ }^{\mathrm{b}}(2-5) 3$ & 1.704 & $0.088 \mathrm{~ns}$ & 0.322 \\
\hline Week 3 & $4(2-5)^{\mathrm{c}}$ & ${ }^{\mathrm{c}}(2-5) 4$ & 1.421 & $0.155 \mathrm{~ns}$ & 0.269 \\
\hline Day 42 & $5(2-5)^{\mathrm{d}}$ & ${ }^{\mathrm{d}}(2-5) 5$ & 0.739 & $0.460 \mathrm{~ns}$ & 0.140 \\
\hline p-value & $<0.001$ & $<0.001$ & &
\end{tabular}

*; significant $(p<0.05) \quad n s ;$ non-significant $(p>0.05)$ 
On comparing the changes in the soft tissue healing of the palatal wound between the two experimental groups throughout the study period, it was observed that there was no statistically significant difference between the two treatment modalities at day 3 , week 2, week 3 as well as day 42 with $(\mathrm{P}=$ $0.678,0.088,0.155$ and 0.460$)$ respectively. However, there was a statistically significance difference between the two treatment modalities at week 1 in favor of PRF group $(\mathrm{P}=.002 *)$

\section{DISCUSSION}

Hyaluronic acid (HA) has a numerous physiological functions such as; anti-inflammatory properties, anti-edematous, antioxidant, bacteriostatic, acceleration of the wound healing, prevention of scar formation and enhancement of tissue regeneration through its ability to retain large amount of water. HA has been chosen as a point of study in many clinical trials due to its feasibility in the form of either gel or spray, also it is a non-invasive method for the patient that is easy to use. Therefore, HA was regarded that it might be a useful dressing for treatment of the palatal wound following the harvesting of FGG (Dahiya, 2013). In this clinical trial $0.2 \%$ HA gel was used since it was found to be more efficient than $0.8 \%$ regarding the acceleration of the palatal wound healing as reported by (Ylldırım et al., 2017).

At the third day post-surgically in the HA group, the periodontal pack was removed and reapplication of $0.2 \% \mathrm{HA}$ gel was performed followed by repacking of the palatal wound by a new noneugenol periodontal pack again. This was done for the purpose of evaluating the healing outcome of the palatal wound and for renewal of the HA gel in situ for better retention over the wound as recommended by the manufacture instruction. On the other hand, in the PRF group the palatal wound was repacked only then, finally the periodontal pack was removed after one week in both groups. (Ross, 2004 and Keceli et al., 2015).
PRF is a platelet concentrate that provides a threedimensional fibrin network rich in growth factors such as fibroblast growth factor (FGF), vascular endothelial growth factor (VEGF), platelet-derived growth factor (PDGF). Therefore, PRF was found to have several advantages especially when used as a palatal bandage in promoting wound healing and angiogenesis (Choukroun, 2001).

The post-operative pain following harvesting of FGG starts during the initial phase of the palatal wound healing (0-3 days) and decreases throughout the second phase (4-10 days) till it disappears at the third phase (11-42 days). Therefore, the assessment of the postoperative pain in this study was limited only to the first week post-operatively, while the assessment of the palatal wound healing was continued up to 42 days since the palatal wound needed up to 42 days to be completely covered by a mature keratinized epithelial layer (Burkhardt et al., 2015).

The results of this trial showed that $0.2 \% \mathrm{HA}$ gel could reduce the postoperative pain starting from day 3 as the highest pain score was found at day 2 with median (range) equals $5(0-10)$ till it reached the lowest pain score at day 7 with median (range) equals $0(0-10)$. Although there was a reduction in pain score after 7 days for each group, there was no statistically significant difference in pain reduction between days $1,2,3,4,5,6$ and 7 . This is consistent with the results displayed by Hassan et al., (2021) who showed that the application of $0.2 \% \mathrm{HA}$ in the form of gel reduced the post-operative pain up to 1 week after harvesting FGG.

The results of the current study are inferior to those reported by the randomized controlled clinical trial performed by Yıldırım et al., (2017) who reported less pain scores compared to our study with median (range) $1.5(0-4), 0.5(0-4), 0(0-0)$ and $0(0-0)$ at day 3 then at one, two and three weeks postoperatively respectively reaching a conclusion that $0.2 \% \mathrm{HA}$ offered a statistically significant effect 
in decreasing the post-operative pain from day 3 to day 21. This contrast between the results could be attributed to using eugenol periodontal dressing as the eugenol might have shared the HA gel in decreasing the postoperative pain. In addition, the recorded pain score at day 3 in their study was 1.5 which is still superior to the current study. This may be due to the effect of trauma caused by suturing of the periodontal pack over the HA gel in the present study. This disparity could be also blamed on the time of pain assessment which was recorded over a long term follow up period (3 weeks) while in the current study, the assessment was recorded daily over a short term follow up period (7 days), since post-operative pain following harvesting FGG would occur mainly during the initial and proliferation phases (3-10 days).

In the PRF group of the current study, the pain scores were reduced from day 4 , as the highest pain score was found at day 3 with a median (range) 4 (0-10) till it reached the lowest pain scores at days 6 and 7 with median (range) $0(0-10)$ and $0(0-6)$ respectively. Although there was a reduction in pain scores after 7 days, there was no statistically significant difference in pain reduction $(\mathrm{P}=0.485)$ between days 1, 2, 3, 4, 5, 6 and 7. The results of the case series reported by Kulkarni et al., (2014) coincide with our results. They reported that using PRF as a palatal dressing following harvesting FGG reduced the post-operative pain up to 1 week postoperatively, but the difference was statistically insignificant. The mean pain score after application of PRF was $3.3( \pm 1.42)$ where the lowest pain score was 1 and the highest score was 6 . Moreover, the results of the study conducted by Tunali et al., 2016, who evaluated the effect of prepared PRF in titanium tubes (T-PRF) for management of the palatal wound after harvesting FGG, were closely similar to those recorded in our present study. They recorded a non-statistically significant difference in pain reduction during the first week.
On the other hand, the results reported by Bahammam,(2018) showed some discrepancy from the present trial. The author reported a statistically significant difference in pain reduction after placing PRF on the palatal wound after harvesting the FGG. The reported pain scores were 1.4, 1.2, 0.5, 0, 0, 0 , 0 from day 1 to day 7 respectively. The difference between the results is clear in all time intervals except for days 6 and 7. This could be justified by the intake of $100 \mathrm{mg}$ acetaminophen as prescribed in their study. In addition, there was no repacking visit at day 3 in their study, unlike ours, as the assessment of wound healing was reported every week for 1 month.

Regarding the effect $0.2 \% \mathrm{HA}$ gel on healing of the palatal wound after harvesting FGG, the results of the present study showed that there was a statistically significant difference in the healing outcomes of the palatal wound between (day 3 -week 1) and (weeks 2, 3 and day 42) with a median (range); 2(13) 2(1-3), 3(2-5), 4(2-5), 5(2-5) respectively with no significant difference between day 3 and week 1 and between weeks 2, 3 and day 42. These results are supported by the RCT conducted by Yildırım et al. (2017) showing that $0.2 \%$ HA could improve the healing of the palatal wound with statistically significant difference at week 1 , week 2 , week 3 and 42 days with median (range) 3 (0-5), 5 (3-7), 8 (6-10) and 10 (9-10) respectively using visual analogue scale (VAS) for assessment of the wound healing. In addition, all donor sites demonstrated complete epithelization on week 2 like this current study resembling the appearance of healing score (3).

The PRF group of the present study showed a statistically significant difference in the healing of the palatal wound between day 3, week 1,2, 3 and day 42 postoperatively with median (range) 2(1-2), $3(2-3), 3(2-4), 4(3-5)$ and 5(3-5) respectively. There was no significant difference between weeks 1 and 2 nor between week 3 and day 42. This could mean that the postsurgical healing was improved by week 
1 and the improvement continued from week 3 till day 42. This is consistent with the results reported by Bahammam (2018) who showed that PRF has significantly improved the palatal wound healing after 1,2,3, 4 and 8 weeks. The assessment of the healing of the palatal wound was performed through the assessment of the color match of the wound on the scale from 1 to 4 where the healing score of the palatal wound in PRF group was 3.8, 3.1, 2.1, 1.7, 1.2 at week 1 , week 2 , week 3 , week 4 and week 8 respectively. The author reported that PRF could provide better color, contour and texture of the palatal tissues at all the time intervals.

Furthermore, Sousa et al., (2020) showed similar results through utilizing A-PRF for covering the palatal donor site after harvesting FGG. They claimed reaching an enhanced healing of the palatal wound by a statistically significant in percentage reduction of the wound area with a mean percentage (SD); $2.9 \%$ (10.7), 36.4\% (12.2), 58\% (14.2), 91.5 (14.6) at dayn2, week 1, week 2 and after 30 days.

\section{CONCLUSIONS}

- Both treatment modalities could be successfully used to reduce the postoperative pain and accelerate the palatal wound healing following harvesting of FGG.

- Using $0.2 \%$ HA gel could be considered a simple and less painful procedure for coverage of the palatal wound after harvesting FGG without the need of a sophisticated equipment.

\section{Conflict of interest and source of funding statement:}

The authors declare that they have no conflict of interest. The study was self-funded

\section{REFERENCES}

- Agudio G, Cortellini P, Buti J, Pini Prato G. (Periodontal conditions of sites treated with gingival augmentation surgery compared with untreated contralateral homologous sites: an 18- to 35-year long- term study) J Periodontol. 87:1371-1378. (2016);

- Al-hamed, F. S. et al. «Regenerative Effect of Platelet Concentrates in Oral and Craniofacial Regeneration», (August), 1-14. doi: 10.3389/fcvm.2019.00126. (2019)

- Bahammam, M. A. «Effect of platelet-rich fibrin palatal bandage on pain scores and wound healing after free gingival graft : a randomized controlled clinical trial». Clinical Oral Investigations (2018) .

- Borie, E. et al. «Platelet-rich fibrin application in dentistry : a literature review», 8(5), or. 7922-7929.(2015)

- Bosshardt, D. D. «Soft tissue wound healing around teeth and dental implants», 41, or. 6-22. doi: 10.1111/ jcpe.12206. (2014)

- Buff LR, Burklin T, Eickholz P, Monting JS, Ratka-Kruger $P$. Does harvesting connective tissue grafts from the palate cause persistent sensory dysfunction Apilot study. Quintessence Int. 2009;40:479- 489. (2009)

- Burkhardt R, Hämmerle CH, Lang NP; Research Group on Oral Soft Tissue Biology \& Wound Healing. Self-reported pain perception of patients after mucosal graft harvesting in the palatal area. J Clin Periodontol.;42(3):281-7. doi: 10.1111/jcpe.12357. Epub 2015 Feb 16. PMID: 25544993 Mar. (2015)

- Choukroun, J., Adda, F., Schoeffler, C., Vervelle, A., An opportunity in perioimplantology: the PRF in French. Implantodontie 42, 55-62. (2001)

- Dahiya, P. eta Kamal, R. «Hyaluronic Acid : A Boon in Periodontal Therapy», doi: 10.4103/1947-2714.112473. (2013)

- De Resende Evaluation of clinical outcomes of acellular dermal matrix (ADM) allograft compared with autogenous - Clinical Oral Investigations- - 324-120-31197. (2019).

- Femminella B, Iaconi MC, Di Tullio M, et al .Clinical comparison of platelet rich fibrin and a gelatin sponge in the management of pal- atal wounds following epithelialized free gingival graft harvest: a randomized clinical trial. J Periodontol. 2015;27:1-17.(2015).

- Hassan A, Ahmed E, Ghalwash D, Elarab AE. Clinical Comparison of MEBO and Hyaluronic Acid Gel in the Management of Pain after Free Gingival Graft Harvesting: A Randomized Clinical Trial. Int J Dent.;2021:2548665. Published 2021 Aug 14. doi:10.1155/2021/2548665. (2021). 
- Kulkarni MR, Thomas BS, Varghese JM, et al. Plateletrich fibrin as an adjunct to palatal wound healing after harvesting a free gingival graft: a case series. J Indian Soc Periodontol. 18:399-402. (2014).

- Kuru, B. «Effect of Topically-Applied Hyaluronic-Acid on Pain and Palatal Epithelial Wound Healing : An ExaminerBlind , Randomized , Controlled Clinical Trial», or. 1-14. doi: 10.1902/jop.2017.170105. (2017)

- Landry RG, Turnbull RS, Howley T. Effectiveness of benzydamine $\mathrm{HCl}$ in the treatment of periodontal post-surgical patients. Res Clin Forums 1988;10:105-18. (1988).

- Reddy Priyanka, Rekha R. Koduganti Veeren dranath Efficacy of Low-level Laser Therapy, Hyaluronic Acid Gel, and Herbal Gel as Adjunctive Tools in Gingivectomy Wound Healing: A Randomized Comparative Clinical and Histological Study. (2019).

- Sana Harris, Randall. Clinical Evaluation of 3 Techniques to Augment Keratinized Tissue Without Root Coverage. Journal of periodontology. 72.932-8. 10.1902/ jop.2001.72.7.932. (2001).

- Schulz KF, Altman DG, Moher D, for the CONSORT Group.CONSORT Statement: updated guidelines for reporting parallel group randomized trials. Epub 24 March. (2010).

- Silva CO, de Lima AF, Sallum AW, Tatakis DN. Coronally positioned flap for root coverage in smokers and nonsmokers: Stability of outcomes between 6 months and 2 years. J Periodontol ; 78: 1702-1707. (2007)

- Sirintawat N, Sawang K, Chaiyasamut T, Wongsirichat $\mathrm{N}$. Pain measurement in oral and maxillofacial surgery. J
Dent Anesth Pain Med. Dec;17(4):253-263 . doi: 10.17245/ jdapm.2017.17.4.253. Epub 2017 Dec 28. PMID: 29349347; PMCID: PMC5766084. (2017).

- Sousa Filipa, Vanessa Machado, João Botelho, Effect of A-PRF Application on Palatal Wound Healing after Free Gingival Graft Harvesting: A Prospective Randomized Study.European journal of dentistry. (2020).

- Sullivan HC, Atkins JH. Free autogenenous gingival grafts. 1. Principles of successful grafting. Periodontics);6:5-13. (1968).

- Tunali, Esra ercan Effectiveness and Predictability of Titanium-Prepared Platelet-Rich Fibrin for the Management of Multiple Gingival Recessions .clin.oral.investigation 10.1007/s00784-017-2211-2 (2016)

- Yıldırım, S. et al. «Effect of Topically-Applied Hyaluronic-Acid on Pain and Palatal Epithelial Wound Healing: An Examiner-Blind, Randomized, Controlled Clinical Trial», Journal of Periodontology, or. 1-14. doi: 10.1902/ jop.2017.170105. (2017).

- Zucchelli, G. et al. «Patient morbidity and root coverage outcome after subepithelial connective tissue and de-epithelialized grafts: a comparative randomizedcontrolled clinical trial», 728-738. doi: 10.1111/j.1600051X.2010.01550. (2010).

- Zucchelli, G. et al. «Autogenous soft tissue grafting for periodontal and peri-implant plastic surgical reconstruction 1 PERIODON TA L A N D AU TOGENOUS SOFT TISSUE», (July), or. 1-8. doi: 10.1002/JPER.19-0350. (2010) 